\title{
Red-Shift Effects in Surface Enhanced Raman Spectroscopy: Spectral or Intensity Dependence of the Near-Field?
}

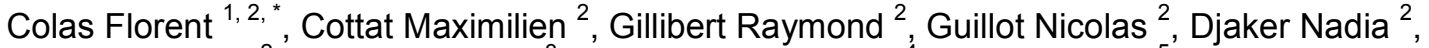 \\ Lidgi-Guigui Nathalie $^{2}$, Toury Timothée ${ }^{3}$, Barchiesi Dominique ${ }^{4}$, Toma Andrea ${ }^{5}$, Di Fabrizio Enzo ${ }^{6,7}$, \\ Gucciardi Pietro Giuseppe ${ }^{8}$, De La Chapelle Marc Lamy ${ }^{2}$
}

${ }^{1}$ IFREMER, Detection, Sensors and Measurements Laboratory, Technology Research and Development Department, F-29280 Plouzané, France

${ }^{2}$ Université Paris 13, Sorbonne Paris Cité, Laboratoire CSPBAT, CNRS, (UMR 7244), 74 rue Marcel Cachin, F-93017 Bobigny, France

${ }^{3}$ ICD-LNIO, UMR STMR CNRS 6279, Université de technologie de Troyes, 12 rue Marie Curie, F10000 Troyes, France

${ }^{4}$ Project Group for Automatic Mesh Generation and Advanced Methods, Gamma3 project (UTT-INRIA), Université de technologies de Troyes, 12 Rue Marie Curie, F-10000 Troyes, France

${ }^{5}$ Istituto Italiano di Tecnologia. Via Morego, 3016163 Genova, Italy

${ }^{6}$ KAUST, King Abdullah University of Science and Technology, Physical Science and Engineering and BESE Divisions, Thuwal, 23955 -6900, Kingdom of Saudi Arabia

${ }^{7}$ University of Magna Graecia, BIONEM lab, Campus Salvatore Venuta, Viale Europa 88100 Germaneto-Catanzaro, Italy

${ }^{8}$ CNR IPCF Istituto per i Processi Chimico-Fisici, Viale F. Stagno D’Alcontres 37, I-98156, Messina, Italy

*Corresponding author : Florent Colas, email address : Florent.Colas@ifremer

\begin{abstract}
:
Optimum amplification in Surface Enhanced Raman Scattering (SERS) from individual nanoantennas is expected when the excitation is slightly blue-shifted with respect to the Localized Surface Plasmon Resonance (LSPR), so that the LSPR peak falls in the middle between the laser and the Stokes Raman emission. Recent experiments have shown when moving the excitation from the visible to the nearinfrared that this rule of thumb is no more valid. The excitation has to be red-shifted with respect to the LSPR peak, up to $80 \mathrm{~nm}$, to obtain highest SERS. Such discrepancy is usually attributed to a Near-Field (NF) to Far-Field (FF) spectral shift. Here we critically discuss this hypothesis for the case of gold nanocylinders. By combining multi-wavelength excitation SERS experiments with numerical calculations, we show that the red-shift of the excitation energy does not originate from a spectral shift between the extinction (FF) and the near-field distribution (NF), which is found to be not larger than $10 \mathrm{~nm}$. Rather, it can be accounted for by looking at the peculiar spectral dependence of the near-field intensity on the cylinders diameter, characterized by an initial increase, up to $180 \mathrm{~nm}$ diameter, followed by a decrease and a pronounced skewness.
\end{abstract}


Introduction:

Surface Enhanced Raman Scattering (SERS) is a powerful technique to detect and characterize chemical $^{1-7}$ or biological ${ }^{8-12}$ species. Due to the high enhancement factor that can be reached in SERS, it has already been demonstrated that molecules can be detected at very low concentrations paving the way to the single molecule sensitivity ${ }^{13-19}$. SERS exploits the optical properties of metallic nanostructures and their resonant interaction with light. It is mainly based on two mechanisms: (i) a chemical ${ }^{20,21}$ and (ii) an electromagnetic ${ }^{22,23}$ effect. The former results from substrate-adsorbate-photon interactions ${ }^{24}$. The enhancement factor from chemical effect is estimated to be of the order of $100^{25,26}$. The second effect is due to the high enhancement of the electromagnetic field at the vicinity of the nanostructure surface. This Near-Field (NF) enhancement is directly related to the excitation of the Localized Surface Plasmon Resonance (LSPR), which is associated to the collective oscillation of the free electron cloud inside the nanostructure. The LSPR is associated to a high electromagnetic field close to the nanostructure. The electromagnetic enhancement can reach up to $10^{8}$ and is then considered as the predominant contribution $^{27,28}$. Such a giant amplification factor comes from a two-step process in which both the excitation and the Raman scattered light are enhanced ${ }^{29,32}$.

The SERS intensity can be written ${ }^{32}$ :

$$
\mathrm{I}_{\mathrm{SERS}}=\mu_{\mathrm{S}} \mathrm{G} \mathrm{M}\left(\lambda_{\text {LASER }}\right) \mathrm{M}\left(\lambda_{\text {Raman }}\right) \frac{\partial \sigma \mathrm{RS}}{\partial \Omega} \Delta \Omega_{\text {Det }} S_{\text {inc }}
$$

where $\mu_{\mathrm{S}}$ is the surface density of the molecules on the substrate, $\mathrm{G}$ a geometrical factor (See supplementary information), $\mathrm{M}(\lambda)$ the local field enhancement averaged over the substrate surface at the wavelength $\lambda, \frac{\partial \sigma_{\mathrm{RS}}}{\partial \Omega}$ the differential Raman scattering cross-section, $\Delta \Omega_{\text {Det }}$ the solid angle for detection and $S_{\text {inc }}$ the incident irradiance in the plane of the substrate. 
The close relation between the LSPR and the SERS intensity then depends strongly on the spectral features of the enhancement factor $M(\lambda)^{33-39}$. It has been shown that it is necessary to finely tune the optical properties of the nanostructures in order to reach the highest enhancement factor. Optimization requires maximizing the product of the NF at the excitation and at the Raman band wavelengths (equation 1), which depend strongly on the nanoparticle shape ${ }^{30,35}$ and on the excitation wavelength ${ }^{30,37}$.

Several studies claimed that the best enhancement factor is reached for a LSPR position at the midway between the $\lambda_{\text {LASER }}$ and $\lambda_{\text {Raman }} 29,34,36,37$.

$$
\lambda_{\mathrm{LSPR}}^{\mathrm{Opt}}=\frac{\lambda_{\mathrm{LASER}}+\lambda_{\mathrm{Raman}}}{2}
$$

In former studies ${ }^{38,40}$, it has been suggested that the deviation between the position of the LSPR given by equation 2 and the experimental results are due to spectral shift between the farfield (FF) resonance, i.e. the energy at which maximum extinction occurs, and a more subtle near-field (NF) resonance, i.e. the energy at which the enhanced field experienced by the molecules in the NF of the nanoantennas is maximum. In other words, does the LSPR peak measured in the FF correspond to the maximum of the NF? Many papers have debated on this subject theoretically and experimentally ${ }^{40-48}$. It has been shown that the NF is generally redshifted compare to the FF ${ }^{40-46}$ and this spectral shift increases with the particle volume ${ }^{44,45}$. However, the cause of this redshift is still being investigated. Recent papers aimed to find phenomenological explanation. Zuloaga and co-workers ${ }^{46}$ demonstrated that this effect comes from the losses by the nanoparticles in analogy with driven damped harmonic oscillator and then demonstrated the universality of this rule. This work was completed by Kats and co-worker ${ }^{47}$ Recently Moreno et al. confirmed the universality of this NF to FF redshift in the case of small particles with a strong dipolar contribution ${ }^{48}$. 
In this paper, we investigate the origin of the wavelength dependence of the SERS enhancement of gold nanocylinders (NCs) by combining extinction spectroscopy, SERS measurement of a probe molecule with multi-wavelength excitation and numerical simulation.

Experimental and Theoretical Methods

This study was performed with gold NCs produced by electron-beam lithography and lift-off techniques $^{36}$ on a microscope glass slide. Their diameters range from $70 \mathrm{~nm}$ to $200 \mathrm{~nm}$ with a $10 \mathrm{~nm}$ step. The distance between the $\mathrm{NC}$ is fixed at $200 \mathrm{~nm}$ in both directions. The gold height is $50 \mathrm{~nm}$ with a chromium adhesion layer of $2 \mathrm{~nm}$.

The samples were cleaned by UV-Ozone process during $30 \mathrm{~min}$, plunged into an ethanol solution and then dried prior to the extinction measurements. The extinction spectra were acquired on a LabRam Xplora spectrometer (Horiba Scientific). The extinction measurements were recorded in transmission configuration with a $10 \mathrm{X}$ objective $(\mathrm{NA}=0.25)$ on an area of $100 \mu \mathrm{m}$ diameter selected by the confocal hole. The sample was illuminated in normal incidence with collimated white light.

The probe molecule was deposited on the nanostructures by immersion in a $10^{-3} \mathrm{M}$ solution of trans-1,2-bis(4-pyridyl)ethylene (BPE) during $1 \mathrm{~h}$ and dried with nitrogen gas flow. The SERS spectra were acquired on three different spectrometers from Horiba Scientific: a LabRam 300 at $633 \mathrm{~nm}$, an Xplora at $660 \mathrm{~nm}$ and $785 \mathrm{~nm}$ and a LabRam HR800 at $691 \mathrm{~nm}$. The signals were collected using a 100x objective with a numerical aperture of 0.9. Acquisition time for one spectrum was 60 s with $200 \mu \mathrm{W}$ power. For each diameter, measurements were done nine times on different points to analyze the reproducibility of the signal. SERS signals were then averaged and a standard deviation was calculated and represented as the error bars. 
The FF and NF calculation was carried out using DDSCAT $7.3^{49,50}$. Single gold NC and array of gold NCs was considered on top of a layer of N-BK7 glass, a chromium film of $2 \mathrm{~nm}$ thickness in between. The refractive index of gold was taken from Johnson and Chrity ${ }^{51}$, the chromium one from Sopra database and the refractive index of the glass substrate was taken from the Schott database. The NC top angle was rounded with a radius of curvature of $10 \mathrm{~nm}$ to take into account the imperfection of nanoparticles fabrication process. The interdipole length was set to $2 \mathrm{~nm}$ (see supplementary data). The height of the $\mathrm{NC}$ was $50 \mathrm{~nm}$ and the diameters were $100 \mathrm{~nm}, 130 \mathrm{~nm}, 150 \mathrm{~nm}, 180 \mathrm{~nm}, 200 \mathrm{~nm}, 230 \mathrm{~nm}, 260 \mathrm{~nm}, 280 \mathrm{~nm}$ and $300 \mathrm{~nm}$. The calculations were performed at wavelength from 500 to $1000 \mathrm{~nm}$ with $50 \mathrm{~nm}$ steps far from the resonance and with $25 \mathrm{~nm}$ step close to the resonance. They were performed with the high performance computing facilities of Pôle de Calcul Intensif pour la Mer ${ }^{52}$. The substrate was incorporated in the object as a slide of glass of $100 \mathrm{~nm}$. This choice is described and motivated in the supplementary data.

The NF enhancement was calculated by averaging the electric field in a volume of $2 \mathrm{~nm}$ thickness around the nanoparticles. The mean SERS enhancement was assessed by the product of the NF enhancement at the laser wavelength and at the vibrational mode wavelength (Supplementary informations). The uncertainties on the position of the FF and NF plasmon bands as well as on the FWHM were assessed from interpolation errors. 
(a)

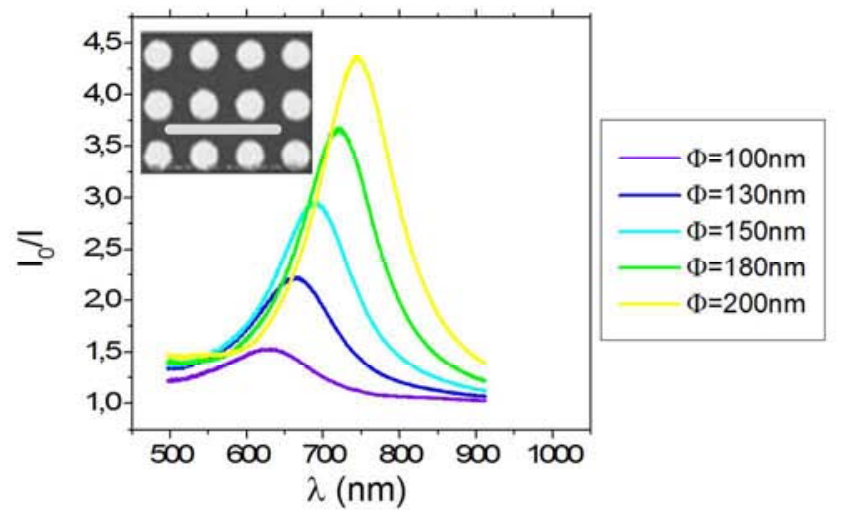

(a)

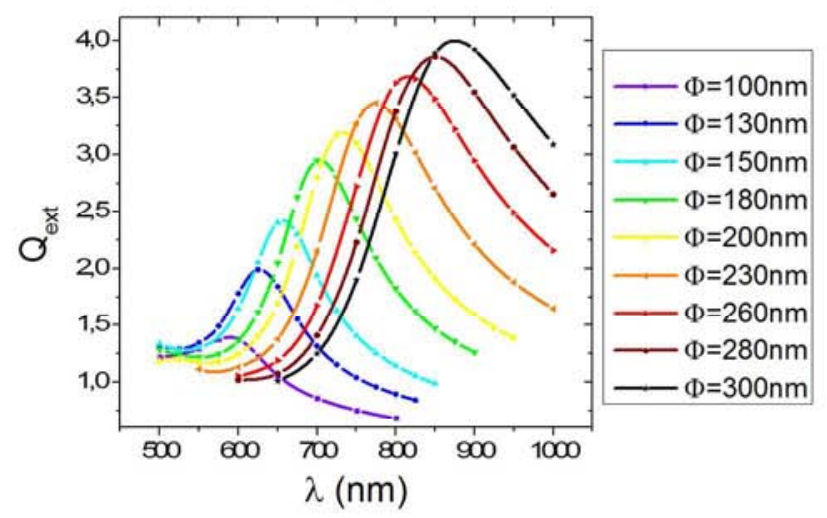

(c)

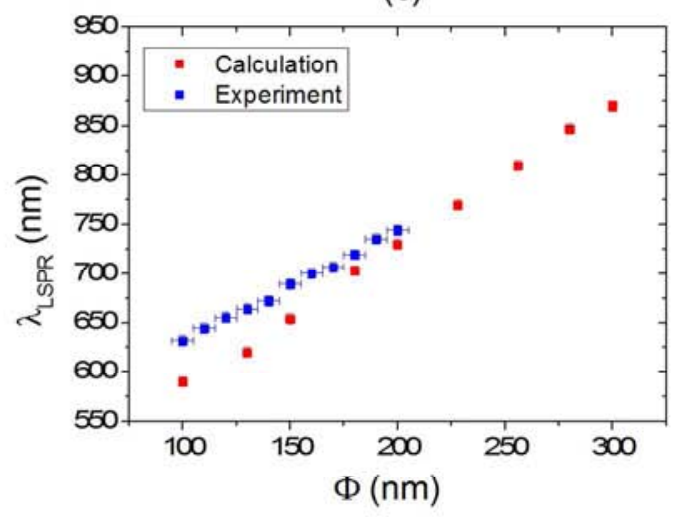

(d)

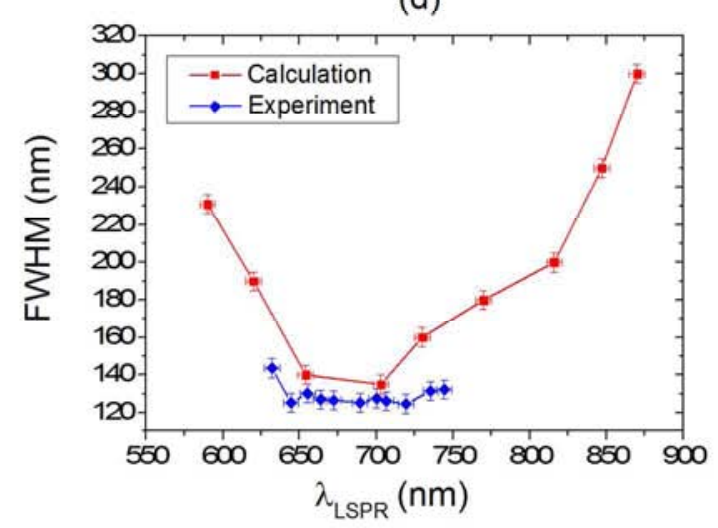

Figure 1: Experimental (a) and theoretical (b) extinction spectra of the NCs. The inset of the curve of figure 1.a) shows a Scanning electron microscopy (SEM) image of the $200 \mathrm{~nm}-$ diameter NC array (the white scale bare is $1 \mu \mathrm{m}$ ). Positions (c) and FWHM (d) of the measured (blue curve) and calculated (red curve) extinction bands.

Results and Discussion

The experimental and theoretical extinction spectra are plotted in figure $1 \mathrm{a}$ and $1 \mathrm{~b}$ respectively. The positions of the extinction bands red shift when the particle diameter increases as previously demonstrated by Zorić et $a l^{53}$. The position of the expected LSPR peaks (figure 1c, blue symbols) show a linear growth similar to the experimental data (figure 1c, blue symbols), although the values are slightly underestimated. This discrepancy is consistent with the 
geometrical uncertainties of the nanostructures geometry and a surface roughness of few $\mathrm{nm}^{54}$. The Full-Width at Half Maximum (FWHM) of the extinction bands are plotted on figure 1d. They appeared constant over the range of particle diameter experimentally studied, meaning a constant damping of the nanoparticle resonance ${ }^{31}$. The Full Width at Half Maximum (FWHM) of the LSPR peaks in experiments are between 7 to $23 \%$ broader than those in simulations. First, the SEM images of the nanostructures showed that the sizes of the nanocylinders are very reproducible from one NC to the other (Fig. 1.a). The diameter distribution is then very sharp. Second, the FWHM of the LSPR peaks is related to the imaginary part of the relative permittivity, which can show some significant changes from one deposit to the other ${ }^{55}$. The overestimation of the FWHM can then be attributed to the gold relative permittivity change.

The U-shape of the theoretical FWHM curve is similar to that reported by Shen et al ${ }^{56}$. The gold absorption is minimal in the $600-700 \mathrm{~nm}$ range ${ }^{51}$, which lead to a resonance sharper in this domain. In addition, as the volume of the NC increases the radiation damping rises and then the resonance peak widens. These two effects contribute to this typical U-shape.

To determine the SERS signal, a probe molecule: the trans-1,2-Bis(4-pyridyl)Ethylen (BPE) was deposited on the NCs. This compound has been extensively used for probing electromagnetic enhancement as the chemical contribution was proven negligible ${ }^{57}$. 


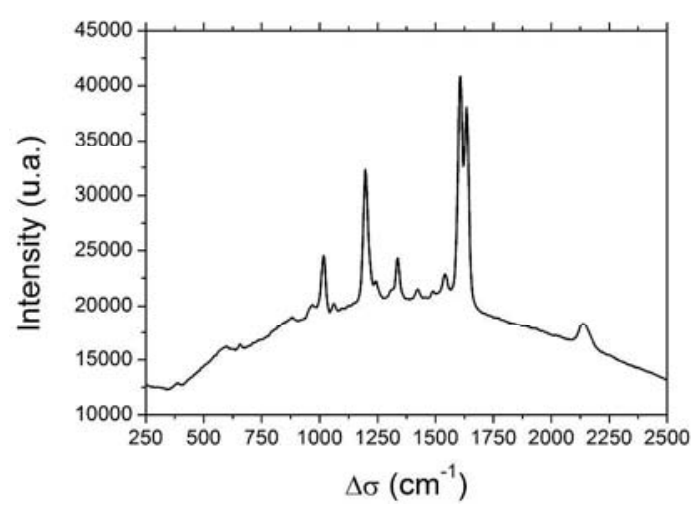

Figure 2: Spectrum of BPE on the $160 \mathrm{~nm}$ diameter NCs excited by the laser at $660 \mathrm{~nm}$.

Figure 2 shows the SERS spectrum of BPE on $160 \mathrm{~nm}$ diameter NCs. The four main peaks at 1007, 1200, 1606 and $1636 \mathrm{~cm}^{-1}$ are easily observed. They are in agreement with the bands previously reported 57,58 . They can be attributed to the ring-breathing mode of pyridine, the $\mathrm{C}=\mathrm{C}$ stretching mode, the aromatic ring stretching mode and the in-plane ring mode, respectively ${ }^{58,59}$. 
(a)

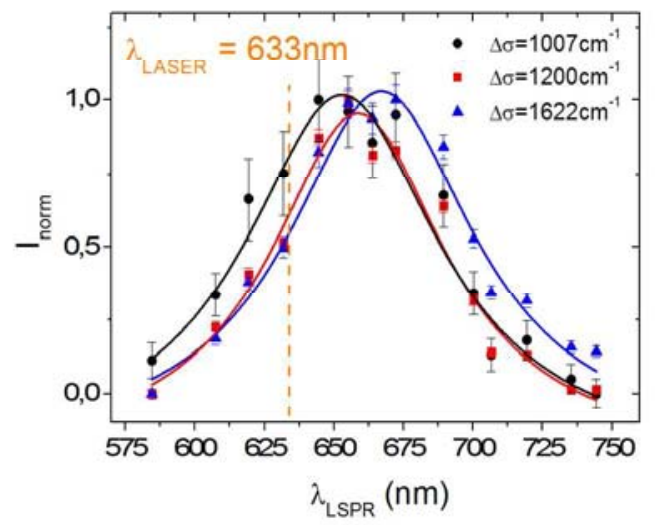

(b)

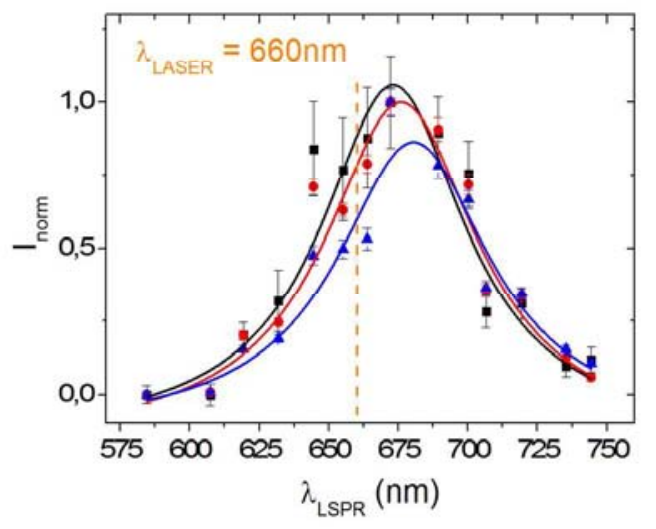

(c)

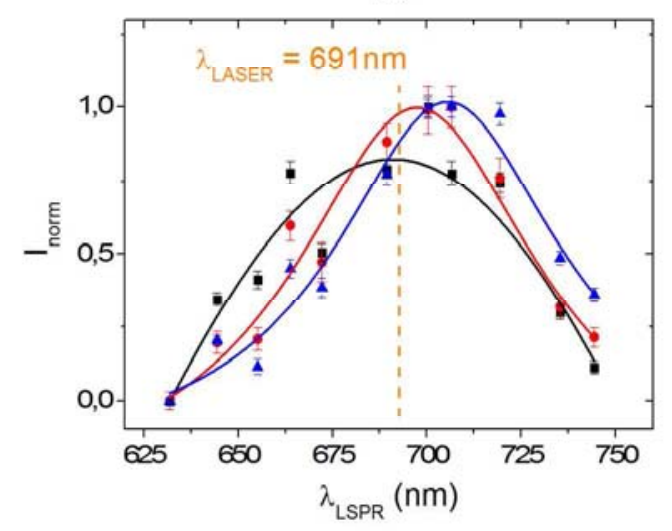

(d)

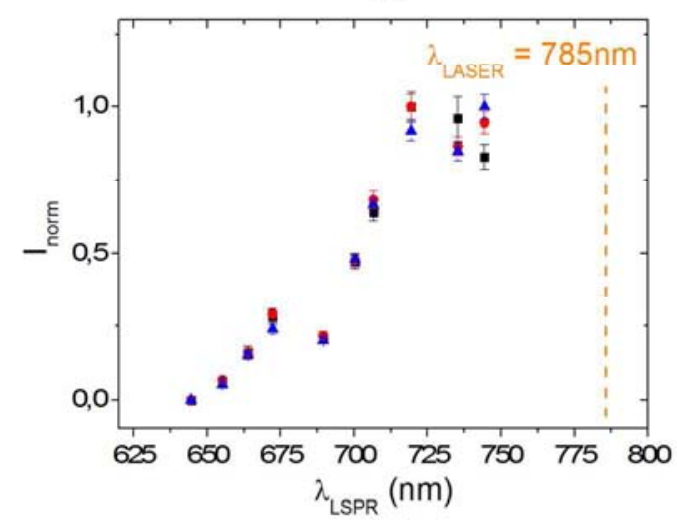

Figure 3: Measured Raman bands intensity at $1007 \mathrm{~cm}^{-1}, 1200 \mathrm{~cm}^{-1}$ and $1622 \mathrm{~cm}^{-1}$ with regards to the LSPR positions for four excitation wavelengths: $633 \mathrm{~nm}(\mathrm{a}), 660 \mathrm{~nm}(\mathrm{~b}), 691 \mathrm{~nm}$ (c) and $785 \mathrm{~nm}(\mathrm{~d})$

Multiwavelength-excitation SERS experiments have been carried out with four different laser wavelengths $(633,660,691$ and $785 \mathrm{~nm})$. The results are plotted in figure 3. For each laser line the SERS intensity of the different Raman modes was plotted as a function of the LSPR wavelength of the NCs. The $1622 \mathrm{~cm}^{-1}$ wavenumber corresponds to the average of the two peaks at 1606 and $1636 \mathrm{~cm}^{-1}$. The SERS intensity of each peak was normalized to its maximum value. The intensity profiles are fitted with lorentzians ${ }^{56}$ to determine the LSPR of the NCs that provide optimal SERS intensity, noted $\lambda_{\mathrm{LSPR}}^{\mathrm{Opt}}$ hereafter. As expected from equation 2 , the larger the 
excitation wavelength, the larger the LSPR wavelength of the nanostructures providing the optimal SERS enhancement (Figure 4)

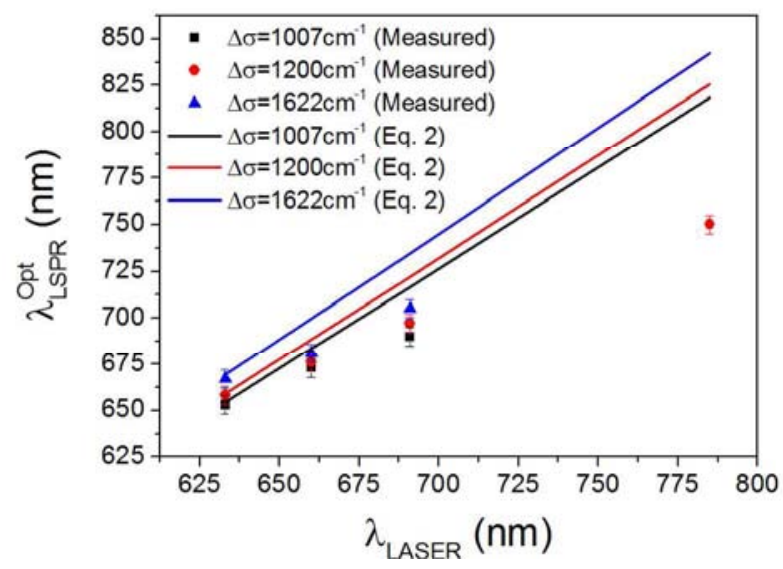

Figure 4: Position of the optimal LSPR wavelengths measured (solid symbols) and expected from eq. 2 (lines) with regards to the excitation wavelength. Data are plotted for all the three Raman bands considered (black for the $1007 \mathrm{~cm}^{-1}$, red for the $1200 \mathrm{~cm}^{-1}$ and green for the 1622 $\mathrm{cm}^{-1}$ doublet. Data for the $785 \mathrm{~nm}$ laser comes from ref 38 .

Tableau 1: Deviation of the expected optimum from Eq. 2 to experimental results for the $1200 \mathrm{~cm}^{-1}$ band.

\begin{tabular}{|l|l|}
\hline$\lambda_{\text {LASER }}(\mathrm{nm})$ & $\Delta \lambda_{1200}(\mathrm{~nm})$ \\
\hline 633 & 1 \\
\hline 660 & 12 \\
\hline 691 & 25 \\
\hline 785 & 80 \\
\hline
\end{tabular}

At $633 \mathrm{~nm}$, the experimental and expected optimal LSPR positions are very similar whereas for larger laser wavelength, the experimental value found for $\lambda_{\mathrm{LSPR}}^{\mathrm{Opt}}$ is gradually blue shifted 
compare to the expected one (Figure 4 and Table 1). In the case of a $785 \mathrm{~nm}$ excitation, we could not reach the maximum of the SERS intensity. However, Guillot et $\mathrm{al}^{38}$ demonstrated that the maximum SERS intensity was achieved at $750 \mathrm{~nm}$ with similar experiment but larger NCs. At this excitation wavelength, the discrepancy of the theoretical to experimental values then goes even up to $80 \mathrm{~nm}$.

The fact that maximum SERS is observed on NCs with a LSPR blue shifted with respect to the expectations has been attributed to a not-well-understood red shift phenomenon between NF and FF as previously debated ${ }^{38,41-48}$. Recently, Zuloaga et $a l .{ }^{46}$ proposed an explanation based on the harmonic oscillator model. The electron cloud of the nanoparticle is described as a damped harmonic oscillator $(\mathrm{HO})$ driven by the electric field of the incoming light. In such a system, two resonances exist: a first one is related to the position of the electron cloud while the second one is related to its speed. Zuloaga et al assimilate the NF resonance to the former while the FF to the latter. They then demonstrate that the pulsations of the NF and the FF resonances, respectively noted $\omega_{\mathrm{NF}}$ and $\omega_{\mathrm{FF}}$, are related by:

$$
\omega_{\mathrm{NF}}=\omega_{\mathrm{FF}} \sqrt{1-\frac{\gamma^{2}}{2 \omega_{\mathrm{FF}}^{2}}}
$$

where $\gamma$ is the damping parameter of the HO.

For low damping, $\gamma \ll \omega_{\mathrm{FF}}$, the relative shift between the wavelength of the NF and FF resonances is expected to be

$$
\omega_{\mathrm{FF}}-\omega_{\mathrm{NF}} \propto \gamma^{2} \lambda_{\mathrm{FF}}
$$

where $\lambda_{\mathrm{FF}}=2 \pi \mathrm{c} / \omega_{\mathrm{FF}}$ that can be measured in far field and corresponds to the $\lambda_{\mathrm{LSPR}}$ and $c$ is the speed of light. 
Several things can be noticed: first, the NF resonance occurs at higher wavelengths with respect to the FF one, i.e. a red-shift is expected in the NF; second, at constant damping, the higher the FF resonance, the higher the expected red-shift; finally, the NF to FF red shift increases with the plasmon damping. The first two predictions from the $\mathrm{HO}$ model seem in qualitative agreement with our experimental findings. 
(a)

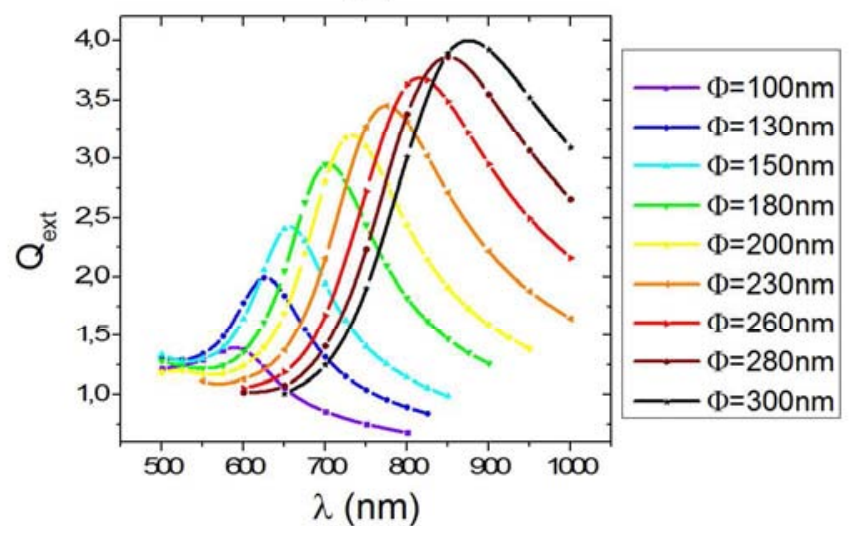

(b)

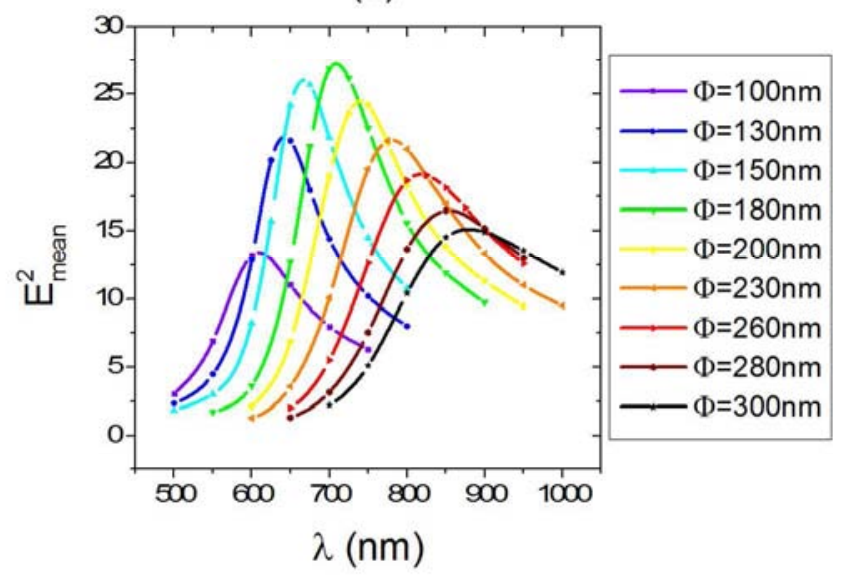

(c)

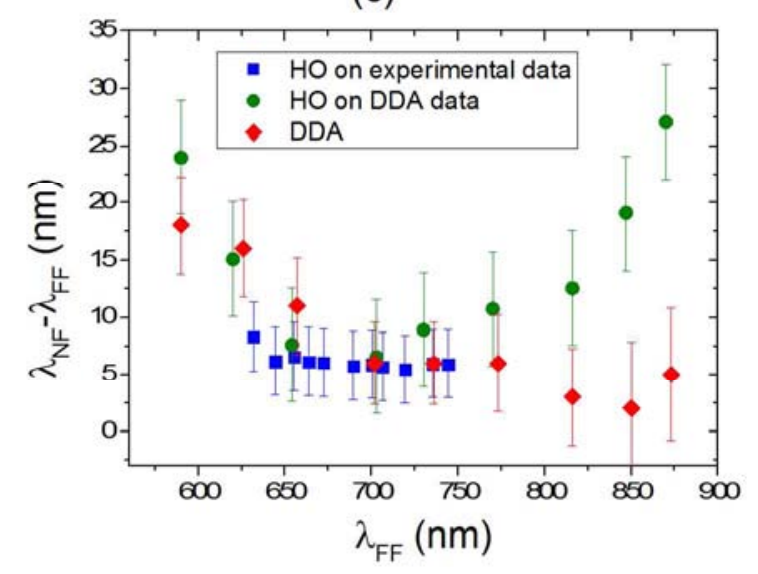

Figure 5 a) Extinction efficiency of arrays of NCs whose diameters vary from 100 to $300 \mathrm{~nm}$ calculated by DDA. b) Mean square electric field around the NCs. c) NF to FF spectral shift 
calculated by the $\mathrm{HO}$ model from experimental (blue square) and calculated (green circle) extinction bands and from near-field calculation (red diamond).

To investigate quantitatively the origin of the red-shift, the spectral dependence of the nearfield distribution for each cylinder diameter was calculated by DDA as the square of the electric field averaged in a $2 \mathrm{~nm}$ thick layer around the nanoparticle. The results are plotted on figure $5 \mathrm{~b}$. The NF to FF shift is shown on figure 5c (red diamonds). For comparison, the expected NF to FF shift was calculated using the HO model. The $\gamma$ term of eq. 3 was estimated from the FWHM of the measured and calculated extinction bands. The results are shown on figure 5c. In the range of the experimental measurement, the NF to FF shift calculated by DDA and HO model are similar and remains lower than $10 \mathrm{~nm}$. For lower resonance wavelengths, NF to FF shift shows an increase while remaining lower than $20 \mathrm{~nm}$. This change is consistent with an increase of the absorption of gold and then of the red shift according to the HO description. For larger resonance wavelengths, the HO model predicts shifts larger that the DDA calculations, but still smaller than $30 \mathrm{~nm}$. Indeed, the model proposed by Zuloaga and co-workers is dedicated to isolated particles small compared to the wavelength and then cannot be applied to larger NCs.

The shift between the optimal positions of the extinction band to the maximum SERS enhancement can reach $80 \mathrm{~nm}$ for larger particles and an excitation wavelength of $785 \mathrm{~nm}$ (Figure 4). Then, we believe that the discrepancy of the experimental data to the equation 2 does not come from a NF to FF spectral shift.

To get more insight on the origin of the discrepancy between the optimal LSPR and the values predicted by equation 2 let's compare the NF of the different NCs (figure 5b). We firstly observe that the NF plasmon bands have some asymmetric profile and positive skewness ${ }^{61}$. Furthermore, the NF intensity increases for diameters from 100 to $180 \mathrm{~nm}$ and then decreases for higher 
diameter, whereas the FF extinction efficiency maximum keeps increasing in this range of particle sizes. The NF intensity gets maximum for an LSPR position around $650-700 \mathrm{~nm}$. In this spectral range, the FWHM of the extinction bands are minimum (figure 1b). The quality factors of the corresponding resonators are then maximum, which lead to the highest near-field ${ }^{56,60}$.

(a)

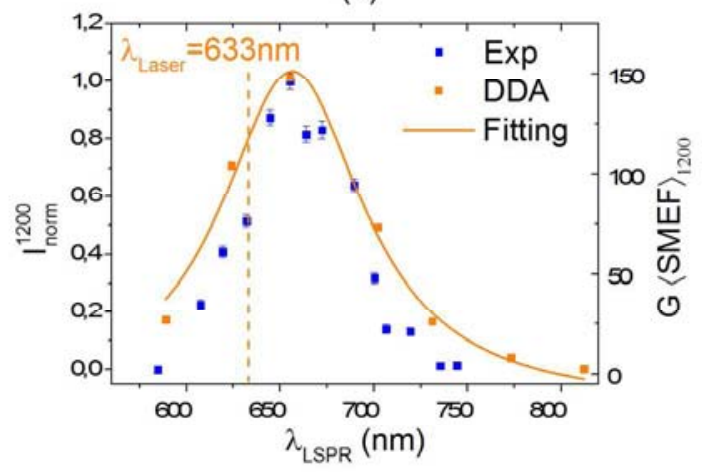

(b)

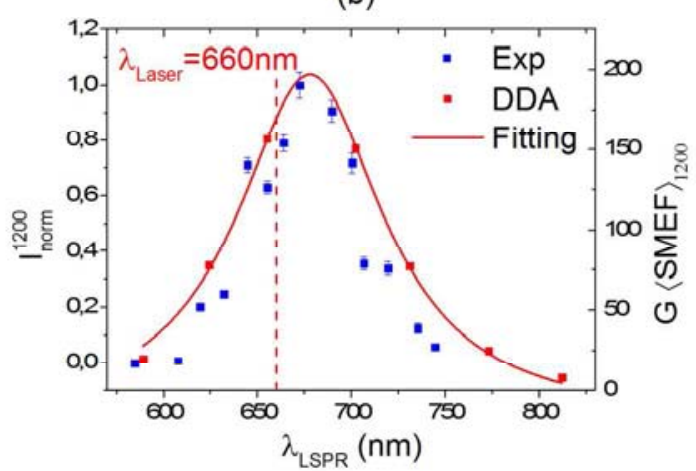

(c)

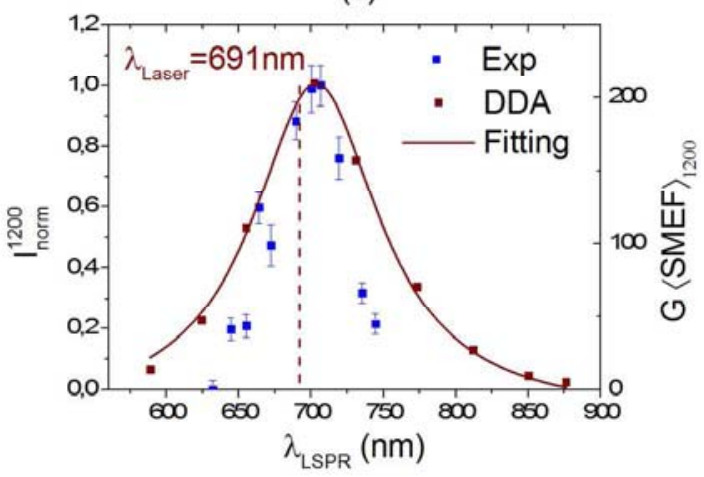

(d)

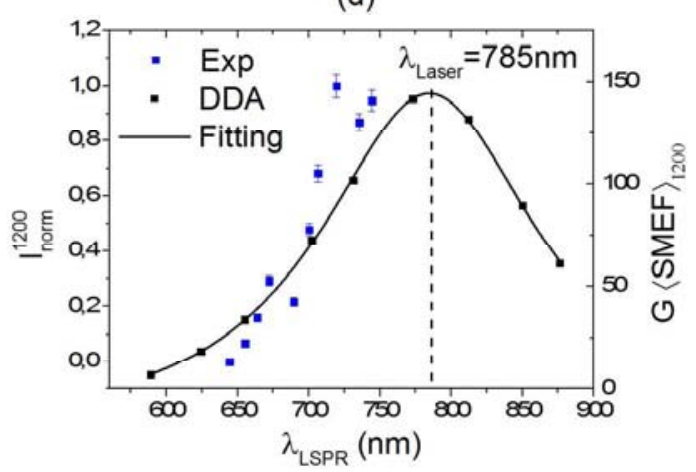

Figure 6: Normalized SERS signal of the $1200 \mathrm{~cm}^{-1}$ band with regards to the extinction band position of the array of NCs measured (on the left hand side y-axis) and the mean SERS enhancement calculated by DDA (on the right hand side y-axis) for the four laser wavelengths: $633 \mathrm{~nm}(\mathrm{a}), 660 \mathrm{~nm}(\mathrm{~b}), 691 \mathrm{~nm}(\mathrm{c})$ and $785 \mathrm{~nm}(\mathrm{~d})$.

The theoretical SERS intensity was calculated from equation 1 (supplementary data) for the four excitation wavelength. The results are plotted on figure 6 and superimposed to the experimental data. The theoretical points were fitted by a lorentzian as Shen and co-workers ${ }^{56}$. 
Theoretical and experimental fit very well for the first three laser line meaning that the enhanced NF calculated by DDA gives an accurate description of the spectral feature of NF as well as the relative intensity. For the $785 \mathrm{~nm}$ laser, the missing experimental points for diameter higher than $200 \mathrm{~nm}$ make the comparison more difficult even if the experimental points are close to the DDA calculations.

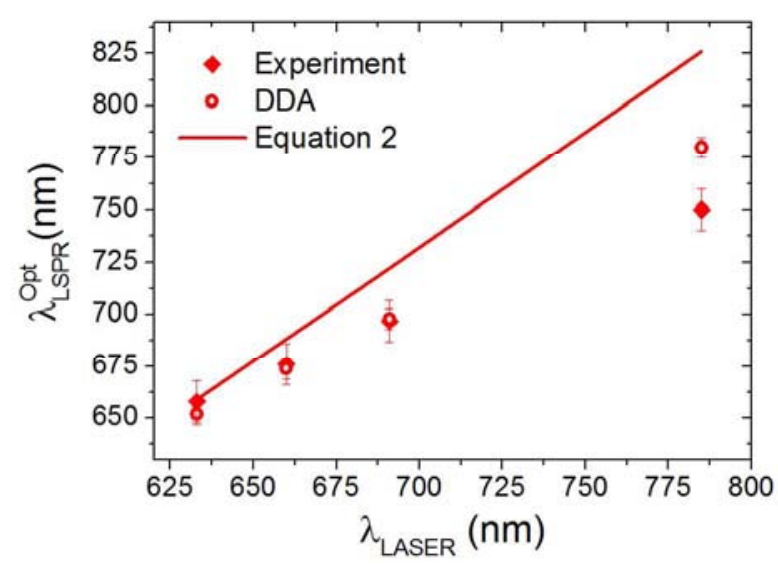

Figure 7: Optimal LSPR position with regards to the excitation wavelength measured (plain markers) and calculated by DDA (shallow markers) and calculated from equation 2 (plain line).

Figure 7 represents $\lambda_{\mathrm{LSPR}}^{\mathrm{Opt}}$ for the $1200 \mathrm{~cm}^{-1}$ Raman shift with regards to the laser wavelengths. The experimental results are enriched by values of previous work done at $785 \mathrm{~nm}^{38}$. Two sets of data are shown: the experimental measurement and the theoretical value calculated from NF DDA simulation. The experimental and theoretical results are very consistent and within the error tolerance except for the $785 \mathrm{~nm}$ laser. Indeed, at this wavelength the theoretical $\lambda_{\mathrm{LSPR}}^{\mathrm{opt}}$ is overestimated by $30 \mathrm{~nm}$ with regards to the experimental one, which is above the error tolerance. This is attributed to the experimental dissimilarities between our study and ref 38 . 


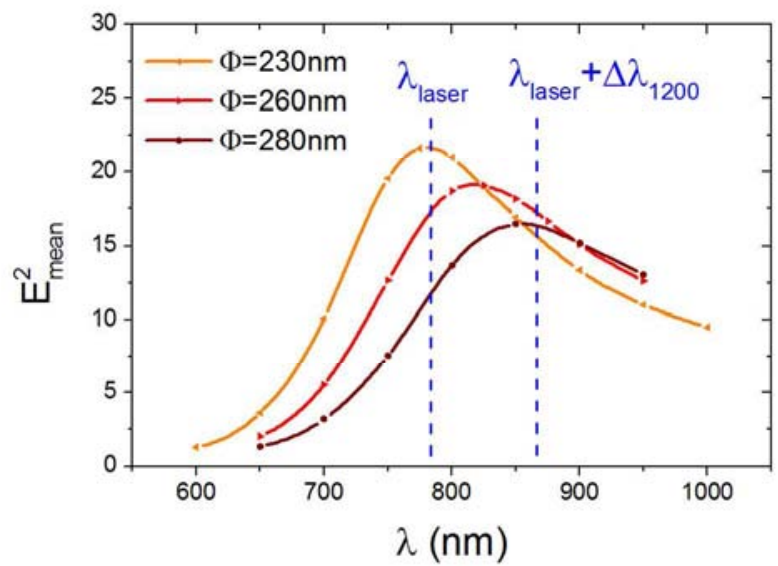

Figure 8: Mean Electric field square around the NC.

Based on this good agreement between the simulation and the experimental data, we propose an explanation for the discrepancy between the optimal extinction band positions measured and calculated with equation 2. Actually, a careful attention has to be paid to the NF intensity and not only to the position of its maximum. For the case of NCs, DDA calculations tell us that the NF intensity gets maximum for an LSPR position around 650-700 nm (figure 6b), corresponding to diameters between 150 and 180nm. Then, as the NC diameter further increases, the intensity of the maximum of the NF intensity decreases. Moreover, the NF curves widen and become asymmetric with a positive skewness increasing with the diameter of the NC. It can be seen on figure 6 that for a laser wavelength of $785 \mathrm{~nm}$, the highest SERS enhancement is then reached if the laser line is close to the maximum of the NF enhancement. This becomes clear when looking at figure 8: the best enhancement is achieved when the intersections of the two dotted lines (the laser of $785 \mathrm{~nm}$ and the Raman shift of $1200 \mathrm{~cm}^{-1}$ wavelength) with the NF plasmon band are the highest. This occurs for the NC of $230 \mathrm{~nm}$ diameter whose plasmon band is maximum at about $780 \mathrm{~nm}$. 
The blue shift experimentally observed does not come from a spectral shift of the NF compare to the FF but from the spectral shape of the NF that is quite different compared to the extinction band (more asymetric).

\section{Conclusions:}

This work characterized the FF and NF properties of gold NCs of diameters from 100 to $300 \mathrm{~nm}$ fabricated by electron-beam lithography. The FF properties were investigated by extinction spectroscopy while the NF by SERS measurements of a probe molecule adsorbed on the nanoparticles. DDA simulations were then performed to provide some phenomenological description.

This study aims to provide some explanations on the position of the optimal LSPR for SERS with regard to the laser line. A rule of thumb states that the best SERS enhancement is achieved when the LSPR is located at the midway between the laser and the Raman lines. However, this does not apply to all the samples, in particular for large laser wavelength. This discrepancy was often assumed to come from a NF to FF spectral shift.

This work clearly demonstrated that there is no significant spectral shift between the NF and the FF with arrays of far-field coupled NCs. The discrepancy between the optimal LSPR for SERS measured and expected from the aforementioned rule cannot be attributed to any NF redshift. The explanation lay in the intensity of the NF plasmon band. The NF enhancement is maximum for LSPR about $670 \mathrm{~nm}$ and then decreases with the LSPR position. A higher SERS signal is then achieved with a LSPR close to this value. The optimal position is then a compromise between the position of the Raman and the laser lines compared to the LSPR and the intensity of the NF band. 
ASSOCIATED CONTENT

\title{
Supporting Information:
}

- Modeling parameters and assessment of the results

- Relation between the near-field and the measured SERS intensity

\section{AUTHOR INFORMATION}

Corresponding Author

florent.colas@ifremer.fr

\section{Author Contributions}

The manuscript was written through contributions of all authors. All authors have given approval to the final version of the manuscript.

\section{ACKNOWLEDGMENT}

Authors want to acknowledge the European project Nanoantenna (HEALTH-F5-2009-241818), and the French Research Agency projects PIRANEX (ANR-12-NANO-0016) and REMANTAS (ANR-11-ECOT-0010) project for the financial support. PGG acknowledges University Paris XIII and the MIUR under Project PRIN 2008J858Y7 financial support.

\begin{abstract}
ABBREVIATIONS
NF, Near-field; FF, Far-Field; BPE, trans-1,2-Bis(4-pyridyl)Ethylen; EBL, Electron Beam Lithography, SEM, Scanning electron microscopy; DDA, Discrete Dipole Approximation; NC, Nanocylinder; FWHM, Full-Width at Half Maximum; SERS, Surface Enhanced Raman
\end{abstract}


Scattering, LSPR, Localized Surface Plasmon Resonance, FDTD, Finite Difference Time Domain; HO, Harmonic Oscillator.

\section{REFERENCES}

1. Chaney, S.B.; Shanmukh, S.; Dluhy, R.A.; Zhao, Y.P. Aligned silver nanorod arrays produce high sensitivity surface-enhanced Raman spectroscopy substrates. Appl. Phys. Lett. 2005, 87(3) 031908-031903.

2. Gupta, R.; Weimer, W.A. High enhancement factor gold films for surface enhanced Raman spectroscopy. Chem. Phys. Lett. 2003, 374(3-4) 302-306.

3. Rodríguez-Lorenzo, L.; Álvarez-Puebla, R.A.; Pastoriza-Santos, I.; Mazzucco, S.; Stéphan O.; Kociak, M.: Liz-Marzán L.M.; García de Abajo, F.J. Zeptomol detection through controlled ultrasensitive surface-enhanced Raman scattering. J. Am. Chem. Soc. 2009, 131(13) 4616-4618.

4. Xu, H.; Bjerneld, E.J.; Käll, M.; Börjesson, L. Spectroscopy of single hemoglobin molecules by surface enhanced Raman scattering. Appl. Phys. Lett. 1999, 83(21), 4357-4360.

5. Michaels, A.M.; Nirmal, M.; Brus, L.E. Surface enhanced Raman spectroscopy of individual rhodamine 6G molecules on large Ag nanocrystals. J. Am. Chem. Soc. 1999, 121(43), 99329939.

6. Qian, X.M.; Nie, S.M. Single-molecule and single-nanoparticle SERS: from fundamental mechanisms to biomedical applications. Chem. Soc. Rev. 2008, 37(5) 912-920.

7. Guillot, N.; Lamy de la Chapelle, M. Lithographied nanostructures as nanosensors. J. Nanophoton. 2012,6 (1) 064506-1-064506-28. 
8. Kneipp, K.; Kneipp, H.; Kartha, V.B.; Manoharan, R.; Deinum, G.; Itzkan, I.; Dasari R.R. Feld MS: Detection and identification of a single DNA base molecule using surface-enhanced Raman scattering (SERS). Phys. Rev. E 1998, 57(6), 6281-6284.

9. Stewart, S.; Fredericks, P.M. Surface-enhanced Raman spectroscopy of peptides and proteins adsorbed on an electrochemically prepared silver surface. Spectrochim. Acta, Part A 1999, 55(7-8), 1615-1640.

10. Han, X.X.; Zhao, B.; Ozaki, Y. Surface-enhanced Raman scattering for protein detection. Anal. Bioanal. Chem. 2009, 394(7), 1719-1727.

11. David, C.; Guillot, N.; Shen, H.; Toury T.; Lamy de la Chapelle, M.L. SERS detection of biomolecules using lithographed nanoparticles towards a reproducible SERS biosensor. Nanotechnology. 2010, 21(47), 475501.

12. Cottat, M.; D’Andrea, C.; Yasukuni, ; Malashikhina, N; Grinyte, R.; Lidgi-Guigui, N.; Fazio, B.; Sutton, A.; Oudar, O.; Charnaux, N.; et al M. High sensitivity, high selectivity SERS detection of MnSOD using optical nanoantennas functionalized with aptamers. J. Phys. Chem. C. 2015, 119 (27) 15532-15540

13. Haynes, C.L.; McFarland, A.D.; Duyne, R.P.V. Surface-enhanced Raman spectroscopy. Anal. Chem. 2005, 77(17), 338-346.

14. Saute, B.; Narayanan, R. Solution-based direct readout surface enhanced Raman spectroscopic (SERS) detection of ultra-low levels of thiram with dogbone shaped gold nanoparticles. Analyst 2011, 136(3), 527-532. 
15. Alvarez-Puebla, R.A.; Dos Santos, Jr. D.S.; Aroca, R.F. Surface-enhanced Raman scattering for ultrasensitive chemical analysis of 1 and 2-naphthalenethiols. Analyst 2004, 129(12), 1251-1256.

16. Kattumenu, R.; Lee, C.H.; Tian, L.; McConney, M.E.; Singamaneni, S. Nanorod decorated nanowires as highly efficient SERS-active hybrids. J. Mater. Chem. 2011, 21(39) 15218-15223.

17. Nie, S.; Emory, S.R. Probing single molecules and single nanoparticles by surfaceenhanced Raman scattering. Science 1997, 275(5303), 1102-1106.

18. Kneipp, K.; Wang, Y.; Kneipp, H.; Perelman, L.T.; Itzkan, I.; Dasari, R.R.; Feld, M.S. Single molecule detection using surface-enhanced Raman scattering (SERS). Phys. Rev. Lett. 1997, 78(9), 1667-1670.

19. Le Ru, E.C.; Grand, J.; Sow, I.; Somerville, W.R.C.; Etchegoin, P.G.; Treguer-Delapierre, M.; Charron, G.; Félidj, N.; Lévi, G.; Aubard, J. A scheme for detecting every single target molecule with surface-enhanced Raman spectroscopy. Nano Lett. 2011, 11(11), 5013-5019.

20. Otto, A. Surface-enhanced Raman scattering of adsorbates. J. Raman Spectrosc. 1991, 22(12), 743-752.

21. Kambhampati, P.; Child, C.M.; Foster, M.C.; Campion, A. On the chemical mechanism of surface enhanced Raman scattering: Experiment and theory. J. Chem. Phys. 1998, 108(12), 5013-5026.

22. Schatz, G.; Young, M.; Duyne, R. Electromagnetic mechanism of SERS. In: Surfaceenhanced Raman scattering; Kneipp, K.; Moskovits, M.; Kneipp, H.,Eds.; Springer: Berlin Heidelberg, 2006; pp 19-45. 
23. Gersten, J.; Nitzan, A. Electromagnetic theory of enhanced Raman scattering by molecules adsorbed on rough surfaces. J. Chem. Phys. 1980, 73(7), 3023-3037.

24. Persson, B.N.J.; Zhao, K.; Zhang, Z. Chemical contribution to surface-enhanced Raman scattering. Phys. Rev. Lett. 2006, 96(20), 207401.

25. Jiang, X.; Campion, A. Chemical effects in surface-enhanced raman scattering: pyridine chemisorbed on silver adatoms on Rh (100). Chem. Phys. Lett. 1987, 140(1), 95-100.

26. Valley, N.; Greeneltch, N.; Van Duyne, R.P.; Schatz, G.C. A look at the origin and magnitude of the chemical contribution to the enhancement mechanism of surface-enhanced Raman spectroscopy (SERS): Theory and experiment. J. Phys. Chem. Lett. 2013, 4(16), 25992604.

27. Le Ru, E.C.; Blackie, E.; Meyer, M.; Etchegoin, P.G. Surface enhanced Raman scattering enhancement factors: A comprehensive study. J. Phys. Chem. C 2007, 111(37), 13794-13803.

28. Xu, H.X.; Aizpurua, J.; Kall, M.; Apell, P. Electromagnetic contributions to singlemolecule sensitivity in surface-enhanced Raman scattering. Phys. Rev. E 2000, 62(3), 43184324.

29. Zimmerman, F.; Wokaun, A. Giant gold clusters stabilized by triphenylphosphine ligands. Characterization by surface enhanced Raman and reflectance spectroscopy. Molecular Physics 1991, $73(5), 959-72$.

30. Guillot. N.; Lamy de la Chapelle, M.L. The electromagnetic effect in surface enhanced Raman scattering: Enhancement optimization using precisely controlled nanostructures. J. Quant. Spectrosc. Radiat. Transfer 2012, 113(18), 2321-2333. 
31. Wokaun, A. Surface-enhanced electromagnetic processes. Solid State Phys. 1984, 38, 223294.

32. Le Ru, E.C. ; Etchegoin, P.G., Chapter 4 - SERS enhancement factors and related topics. Principles of surface-enhanced Raman spectroscopy. In: Principles of surface enhanced Raman scattering. Elsevier 2009, pp.185-264.

33. Haynes, C.L.; Van Duyne, R.P. Plasmon scanned surface-enhanced Raman scattering excitation profiles. MRS Online Proc. Libr. 2002, 728:null-null.

34. Felidj, N.; Aubard, J.; Levi, G.; Krenn, J.R.; Hohenau, A.; Schider, G.; Leitner, A.; Aussenegg, F.R. Optimized surface-enhanced Raman scattering on gold nanoparticle arrays. Appl. Phys. Lett. 2003, 82(18), 3095-3097.

35. Grand, J.; Lamy de la Chapelle, M; Bijeon, J.-L.; Adam, P.-M.; Vial, A.; Royer, P. Role of localized surface plasmons in surface-enhanced Raman scattering of shape-controlled metallic particles in regular arrays. Phys. Rev. B. 2005, 72(3)033407.

36. Grand, J.; Kostcheev, S.; Bijeon, J.L.; Lamy de la Chapelle, M.L.; Adam, P.M.; Rumyantseva, A.; Lérondel, G.; Royer, P. Optimization of SERS-active substrates for near-field Raman spectroscopy. Synth. Met. 2003, 139(3), 621-624.

37. Billot, L.; Lamy de la Chapelle, M.; Grimault, A.S.; Vial, A.; Barchiesi, D.; Bijeon, J.L.; Adam, P.M.; Royer, P. Surface enhanced Raman scattering on gold nanowire arrays: Evidence of strong multipolar surface plasmon resonance enhancement. Chem. Phys. Lett. 2006, 422(4-6), 303-307. 
38. Guillot, N.; Shen, H.; Frémaux, B.; Péron, O.; Rinnert, E.; Toury, T.; Lamy de la Chapelle, M.L. Surface enhanced Raman scattering optimization of gold nanocylinder arrays: Influence of the localized surface plasmon resonance and excitation wavelength. Appl. Phys. Lett. 2010, 97(2), 023113-023113.

39. McFarland, A.D.; Young, M.A.; Dieringer, J.A.; Van Duyne, R.P. Wavelength-scanned surface-enhanced Raman excitation spectroscopy. J. Phys. Chem. B 2005, 109(22), 1127911285.

40. Grimault, A.S.; Vial, A.; Lamy de la Chapelle, M. Modeling of regular gold nanostructures arrays for SERS applications using a 3D FDTD method. Appl. Phys. B 2006, 84(1-2), 111-115.

41. Ross, B.M.; Lee, L.P. Comparison of near- and far-field measures for plasmon resonance of metallic nanoparticles. Opt. Lett. 2009, 34, 896-898

42. Kelly, K.L.; Coronado, E.; Zhao, L.L.; Schatz, G.C. The Optical Properties of Metal Nanoparticles: The influence of size, shape, and dielectric environment. J. Phys. Chem. B 2003, 107, 668-677.

43. Alonso-Gonzales, P.; Albella, P.; Neubrech, F.; Huck, C.; Chen, J.; Golmar, F.; Casanova, F.; Hueso, L.E.; Pucci, A.; Aizpurua, J.; et al. Experimental verification of the spectral shift between near- and far-field peak intensities of plasmonic infrared nanoantennas. Phys. Rev. Lett. 2013, 110, 203902

44. Quinten, M.Optical Properties of Nanoparticle Systems: MieandBeyond.,Wiley-VCH,2011

45. Bryant, G.W.; Garcia de Abajo, F.J.; Aizpurua, J. Mapping the plasmon resonances of metallic nanoantennas. Nano Lett. 2008, 8, 631-636. 
46. Zuloaga, J; Nordlander, P. On the energy shift between near-field and far-field peak intensities in localized plasmon systems. Nano Lett. 2011, 11, 1280-1283.

47. Kats, M.A ; Yu, N; Genevet, P; Gaburro, Z; Capasso, F. Effect of radiation damping on the spectral response of plasmonic components. Opt. Express 2011, 19(22), 21748-21753.

48. Moreno, F.; Albella, P.; Nieto-Vesperinas, M. Analysis of the spectral behavior of localized plasmon resonances in the near- and far-field regimes. Langmuir 2013, 29, 6715-6721.

49. Draine, B.T.; Flatau, P.J. Discrete-dipole approximation for periodic targets: theory and tests. J. Opt. Soc. Am. A 2008, 25, 2593-2703.

50. Flatau, P.J.; Draine, B. T. Fast near field calculations in the discrete dipole approximation for regular rectilinear grids. Opt. Express 2012, 20, 1247-1252

51. Johnson, P.B.; Christy, R.W. Optical constants of the noble metals. Phys. Rev. B 1976, 6 (12), 4370-4379

52. Pôle de Calcul Intensif pour la Mer, PCIM, http://www.ifremer.fr/pcim_accessed June 6, $\underline{2016)}$.

53. Zorić, I.; Zäch, M; Kasemo, B; Langhammer, C. Gold, platinum, and aluminum nanodisk plasmons: material independence, subradiance, and damping mechanisms. ACS Nano. 2011 26; 5(4) $2535-2546$.

54. Barchiesi, D.; Kessentini, S.; Guillot, N.; Lamy De La Chapelle, M.; Grosges, T. Localized surface plasmon resonance in arrays of nano-gold cylinders: inverse problem and propagation of uncertainties. Opt. Express 2013, 21 (2), 2245-2262. 
55. D. E. Aspnes, D. E.; Kinsbron, E.; Bacon, D. D. Optical properties of Au: Sample effects. Phys. Rev. B 1980 21, 3290.

56. Shen, H.; Guillot, N; Rouxel, J.; Lamy de la Chapelle, M. ;Toury, T. Optimized plasmonic nanostructures for improved sensing activities. Opt. express 2012, 20 (19), 21278- 21290.

57. Felidj, N.; Aubard, J.; Levi, G.; Krenn, J.R.; Hohenau, A.; Schider, G.; Leitner., A; Aussenegg F.R. Controlling the optical response of regular arrays of gold particles for surfaceenhanced Raman scattering. Phys. Rev. B. 2002, 65, 075419.

58. Yang, W.H.; Hulteen, J.C.; Schatz, G.C.; Van Duyne, R.P. A surface-enhanced hyperRaman and surface-enhanced Raman scattering study of trans-1,2-bis(4-pyridyl)ethylene adsorbed onto silver film over nanosphere electrodes. Vibrational Assignments: Experiment and Theory. J. Chem. Phys. 1996, 104, 4313.

59. Sun, G.; Grundmeier, G. Surface-enhanced Raman spectroscopy of the growth of ultra-thin organosilicon plasma polymers on nanoporous $\mathrm{Ag} / \mathrm{SiO} 2$-bilayer films. Thin Solid Films 2006 $515,1266$.

60. Lamy de la Chapelle, M.;Shen, H.; Guillot,N.; Frémaux, B.; Guelorget, B.; Toury, T. New Gold nanoparticles adhesion process opening the way of improved and highly sensitive plasmonics technologies. Plasmonics 2013, 8, 411-415.

61. J. Aizpurua, Garnett W. Bryant, Lee J. Richter, F. J. García de Abajo, Brian K. Kelley, and T. Mallouk Optical properties of coupled metallic nanorods for field-enhanced spectroscopy Phys. Rev. B 71, 235420

Table of Content Graphic 
Near-field
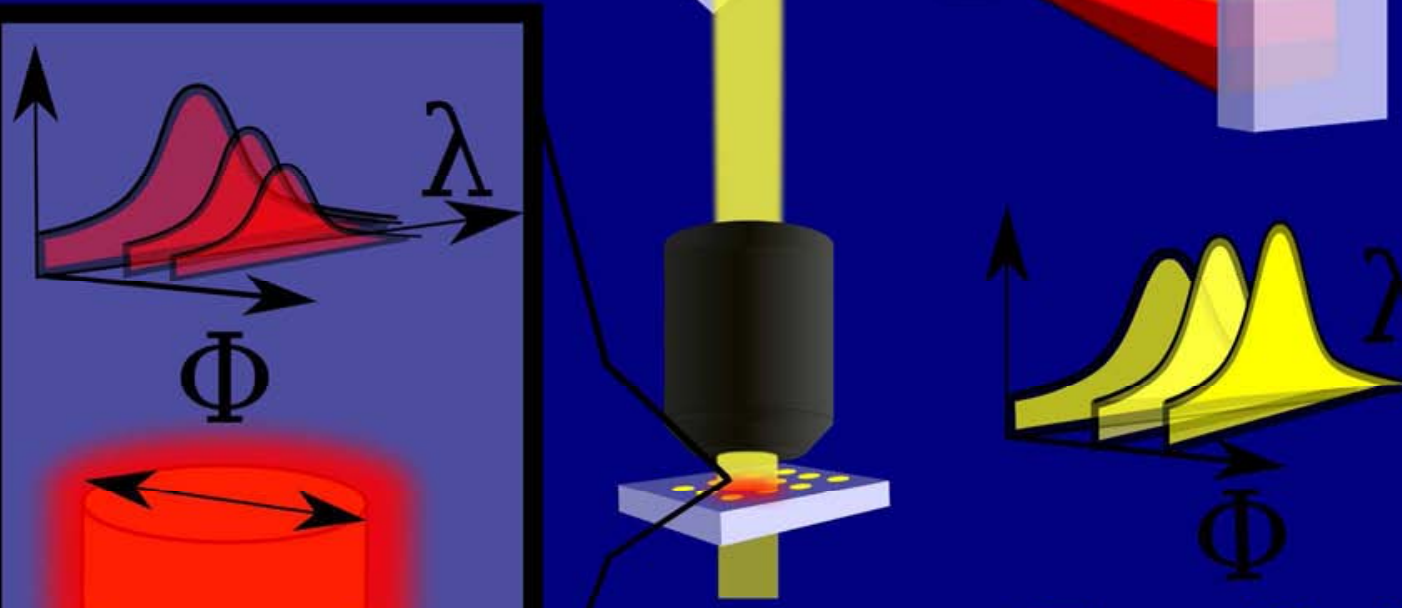

Far-field

Table of content image 
(a)

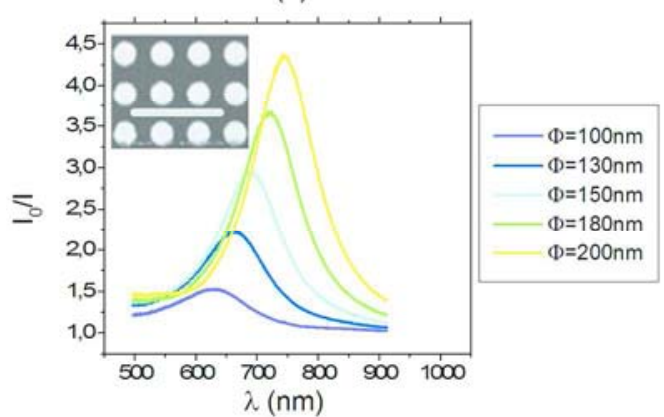

(a)

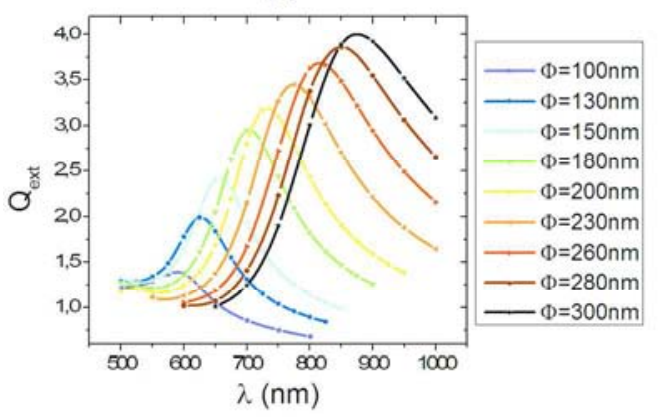

(c)

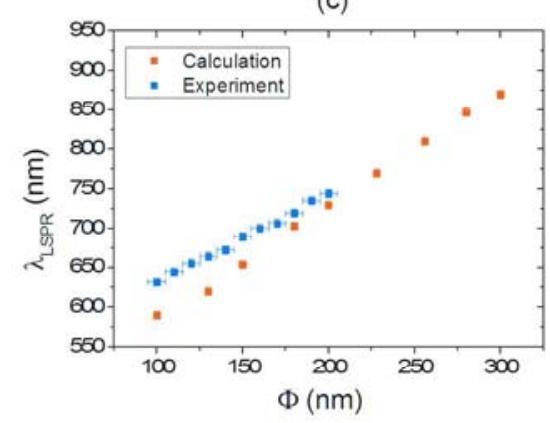

(d)

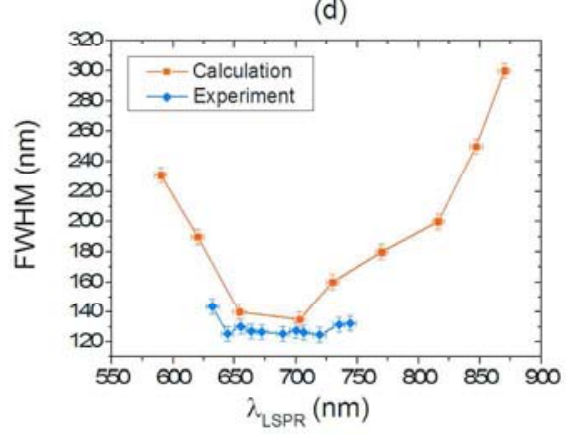

Experimental (a) and theoretical (b) extinction spectra of the NCs. The inset of the curve of figure 1.a) shows a Scanning electron microscopy (SEM) image of the $200 \mathrm{~nm}$ - diameter NC array (the white scale bare is $1 \mu \mathrm{m}$ ). Positions (c) and FWHM (d) of the measured (blue curve) and calculated (red curve) extinction bands.

$177 \times 124 \mathrm{~mm}(300 \times 300 \mathrm{DPI})$ 


1
2
3
4
5
6
7
8
9
10
11
12
13
14
15
16
17
18
19
20
21
22
23
24
25
26
27
28
29
30
31
32
33
34
35
36
37
38
39
40
41
42
43
44
45
46
47
48
49
50
51
52
53
54
55
56
57
59
60

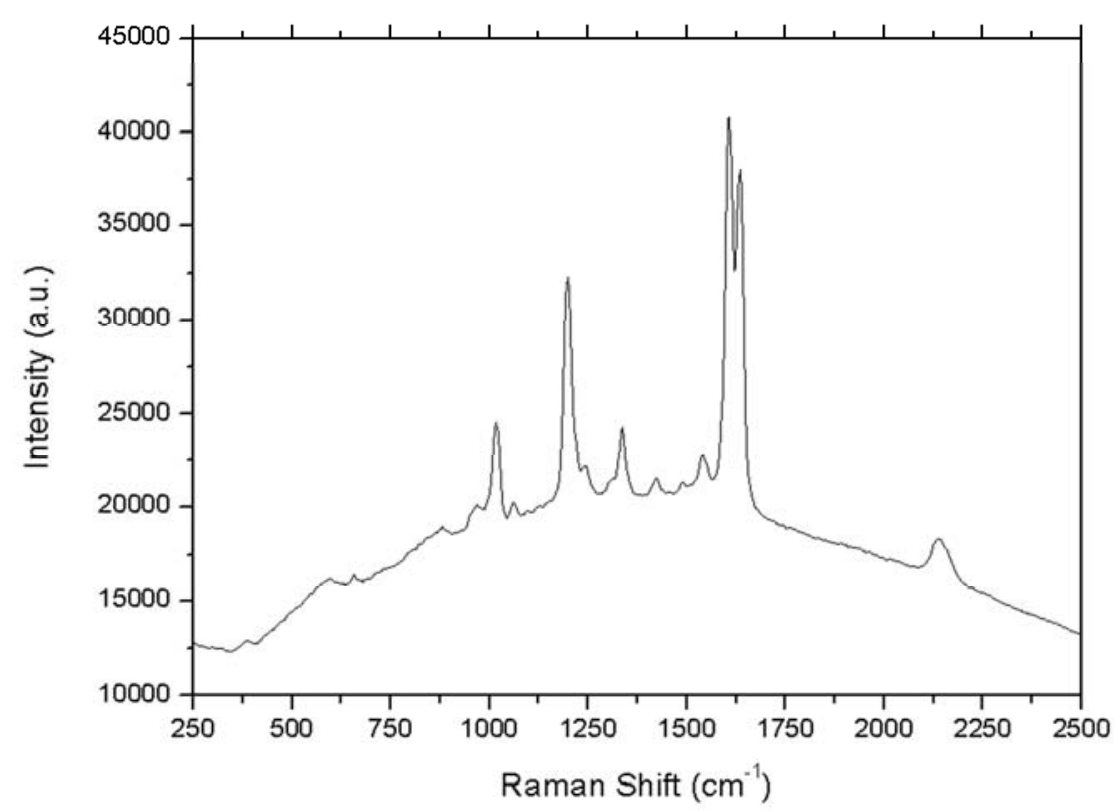

Spectrum of BPE on the $160 \mathrm{~nm}$ diameter NCs excited by the HeNe laser. $88 \times 62 \mathrm{~mm}(300 \times 300 \mathrm{DPI})$ 
(a)

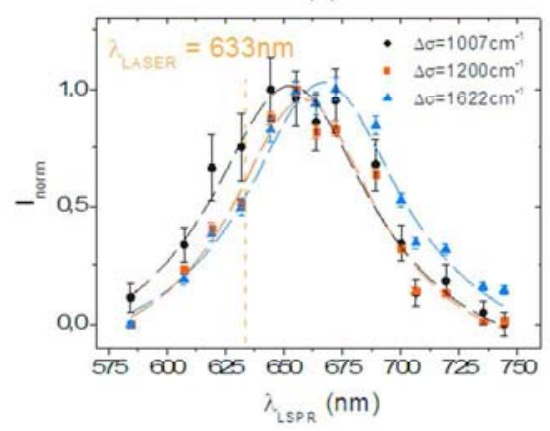

(b)

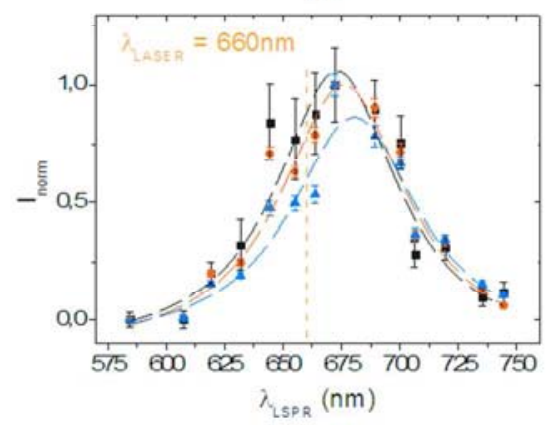

(c)

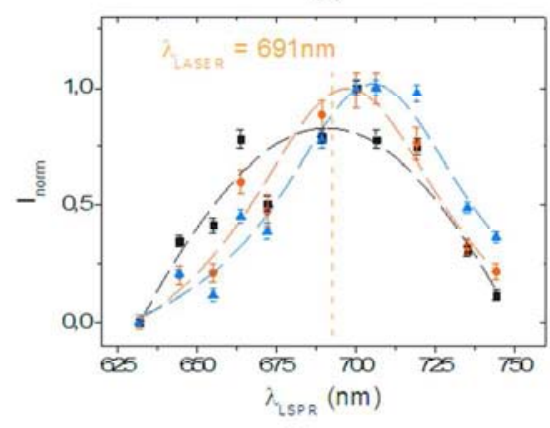

(d)

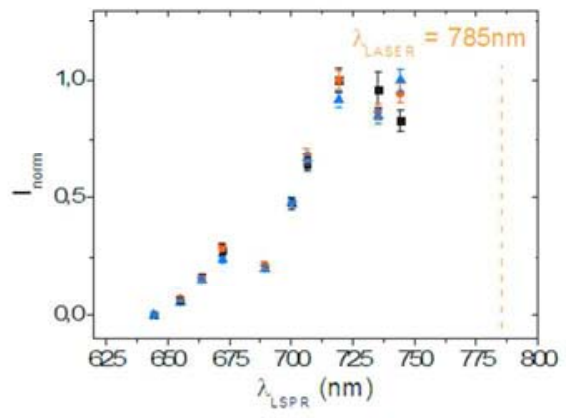

Measured Raman bands intensity at $1007 \mathrm{~cm}^{-1}, 1200 \mathrm{~cm}^{-1}$ and $1622 \mathrm{~cm}^{-1}$ with regards to the LSPR positions for four excitation wavelengths: $633 \mathrm{~nm}$ (a), $660 \mathrm{~nm}$ (b), $691 \mathrm{~nm}$ (c) and $785 \mathrm{~nm}$ (d) $88 \times 63 \mathrm{~mm}(300 \times 300 \mathrm{DPI})$ 


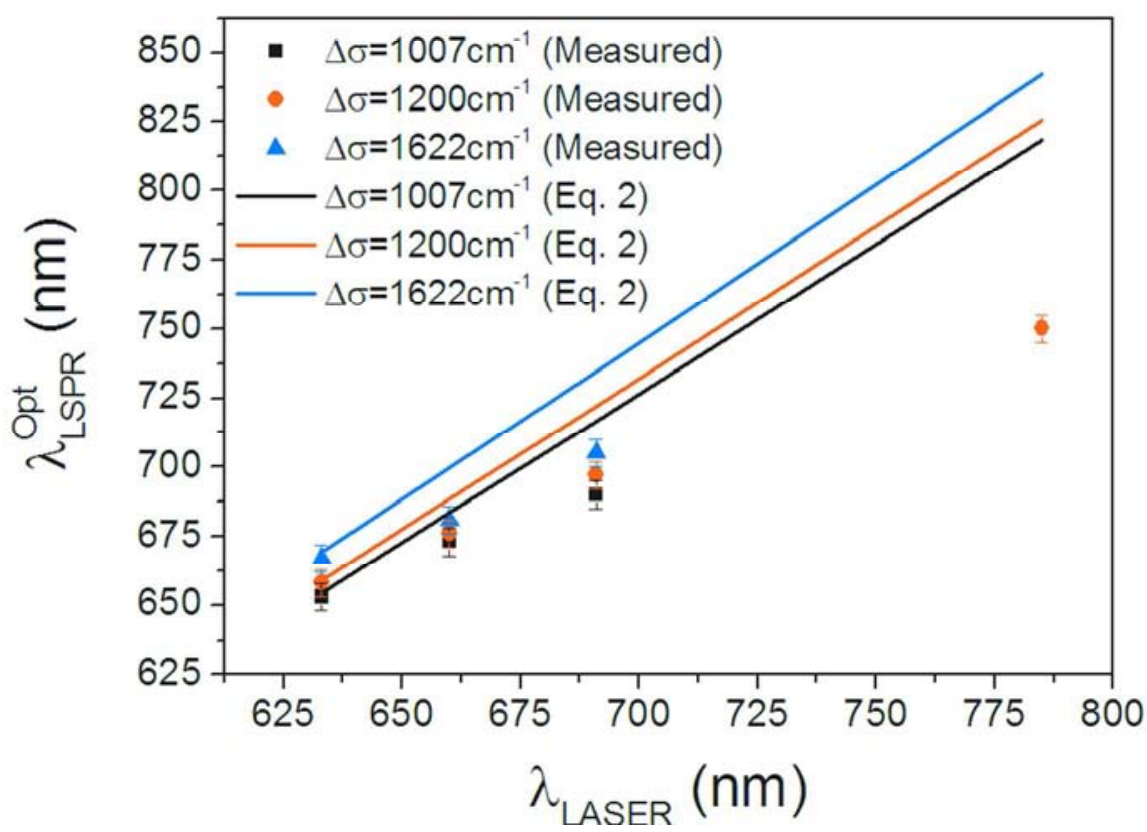

Position of the optimal LSPR wavelengths measured (solid symbols) and expected from eq. 2 (lines) with regards to the excitation wavelength. Data are plotted for all the three Raman bands considered (black for the $1007 \mathrm{~cm}^{-1}$, red for the $1200 \mathrm{~cm}^{-1}$ and green for the $1622 \mathrm{~cm}^{-1}$ doublet. Data for the $785 \mathrm{~nm}$ laser comes from ref 38

$88 \times 62 \mathrm{~mm}(300 \times 300 \mathrm{DPI})$ 
(a)

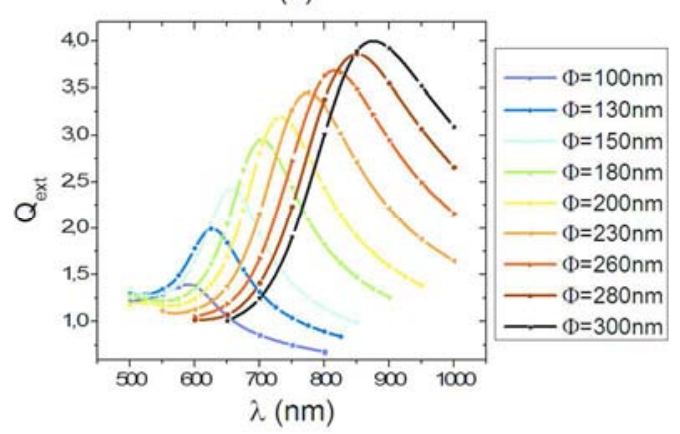

(b)

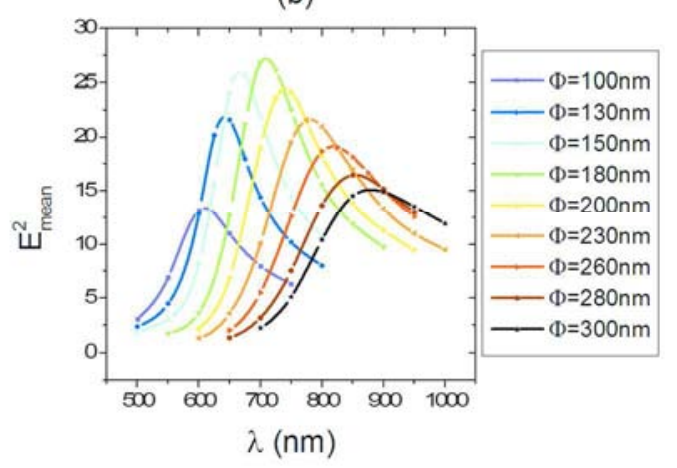

(c)

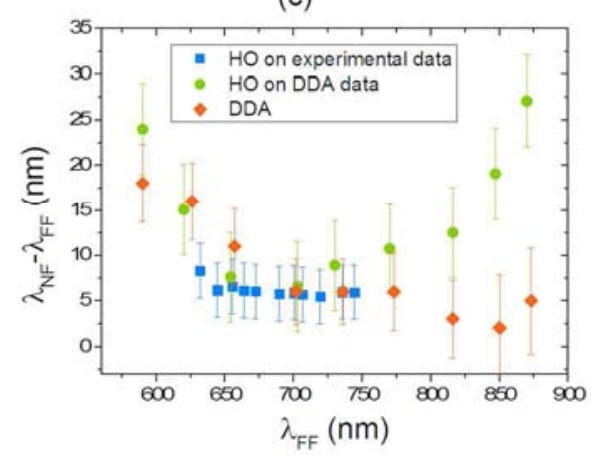

a) Extinction efficiency of arrays of NCs whose diameters vary from 100 to $300 \mathrm{~nm}$ calculated by DDA. b) Mean square electric field around the NCs. c) NF to FF spectral shift calculated by the HO model from experimental (blue square) and calculated (green circle) extinction bands and from near-field calculation (red diamond). $88 \times 200 \mathrm{~mm}(300 \times 300$ DPI $)$ 
(a)

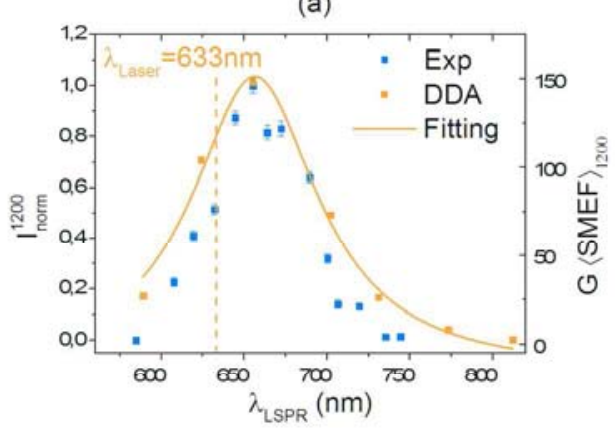

(b)

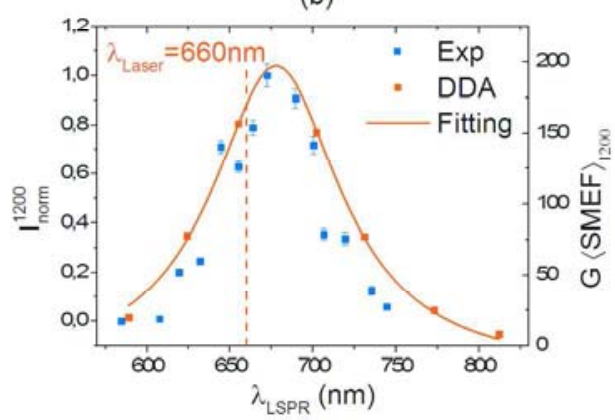

(c)

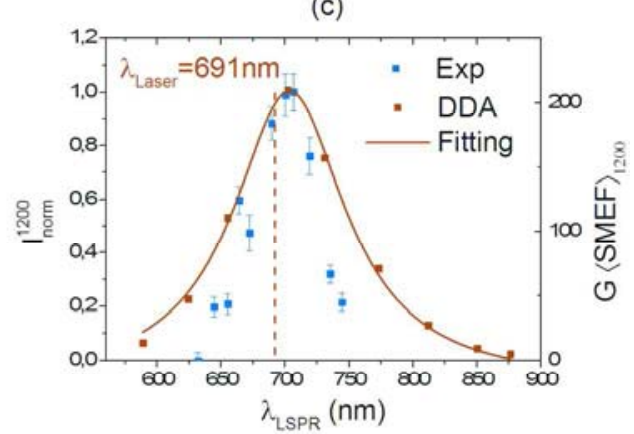

(d)

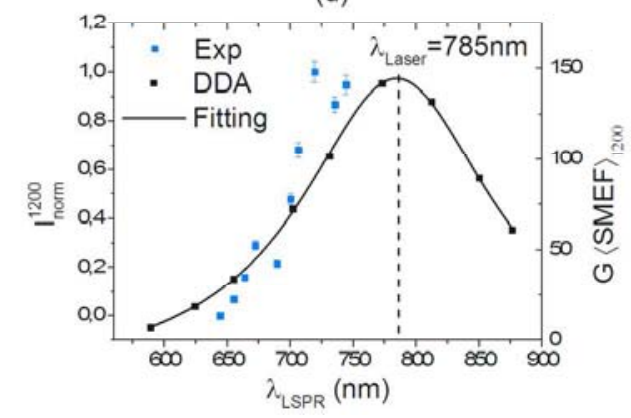

Normalized SERS signal of the $1200 \mathrm{~cm}^{-1}$ band with regards to the extinction band position of the array of NCs measured (on the left hand side y-axis) and the mean SERS enhancement calculated by DDA (on the right hand side y-axis) for the four laser wavelengths: $633 \mathrm{~nm}$ (a), $660 \mathrm{~nm}$ (b), $691 \mathrm{~nm}$ (c) and $785 \mathrm{~nm}$ (d).

$177 \times 124 \mathrm{~mm}(300 \times 300$ DPI $)$ 
Optimal LSPR position with regards to the excitation wavelength measured (plain markers) and calculated by DDA (shallow markers) and calculated from equation 2 (plain line). $88 \times 61 \mathrm{~mm}(300 \times 300 \mathrm{DPI})$ 


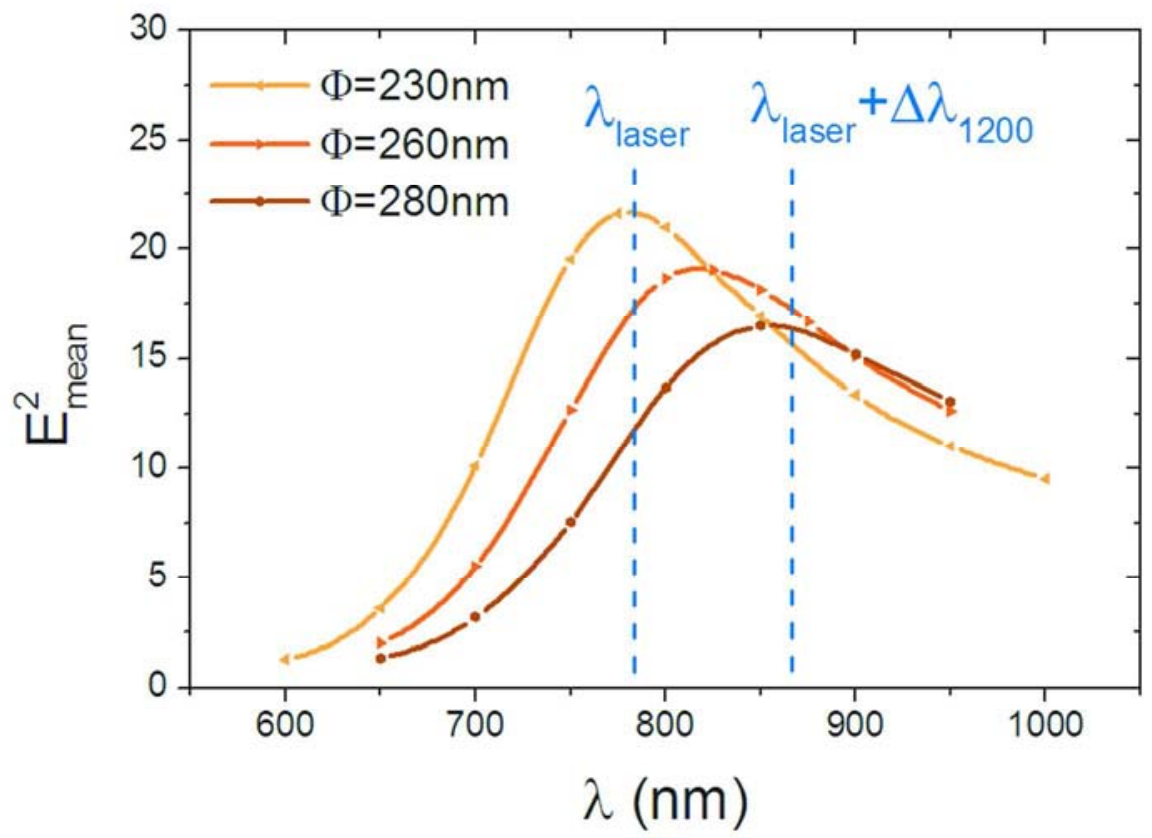

Mean Electric field square around the NC $88 \times 62 \mathrm{~mm}(300 \times 300$ DPI $)$ 


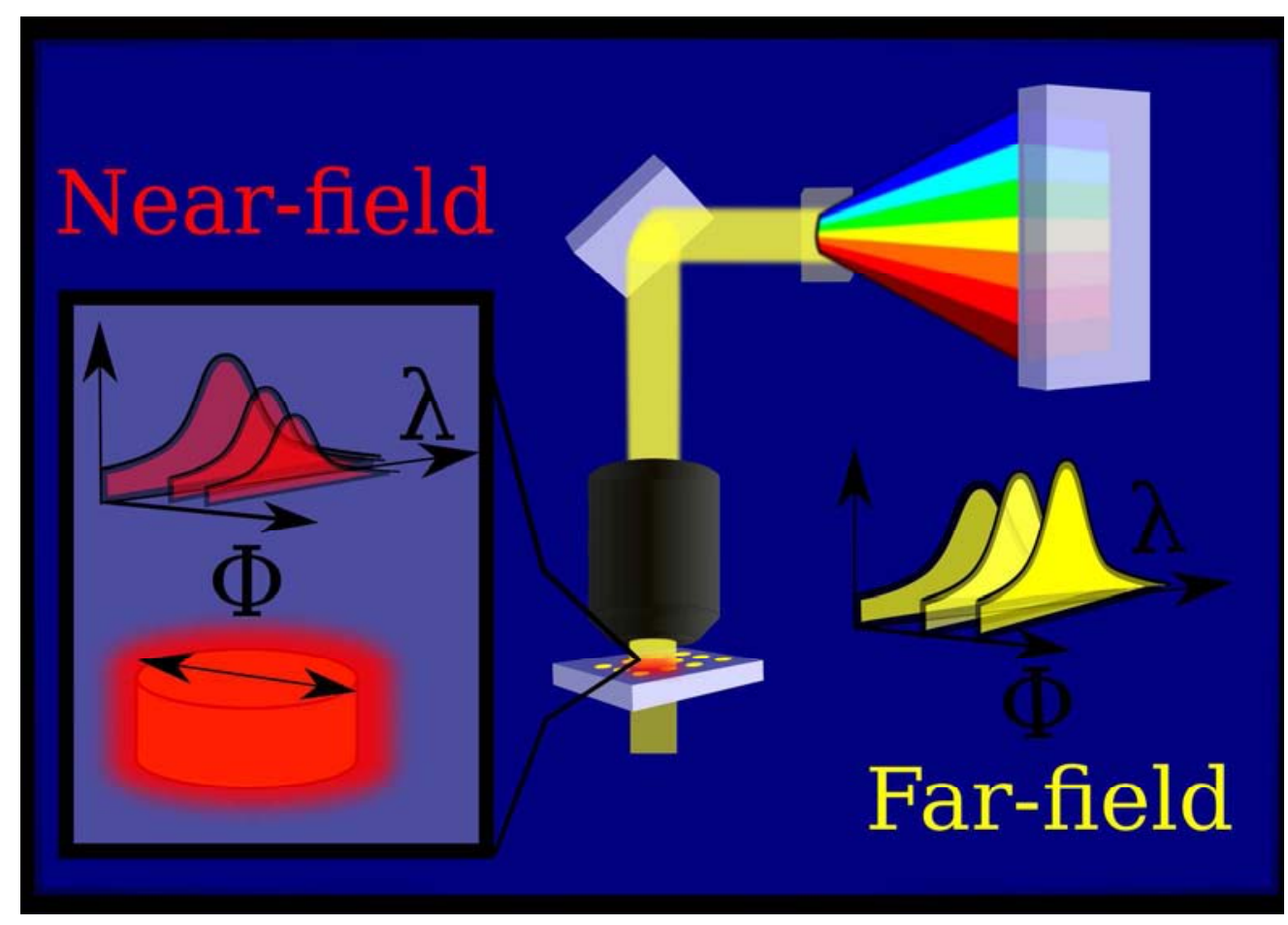

Table of Contents Image $177 \times 126 \mathrm{~mm}(300 \times 300$ DPI $)$ 